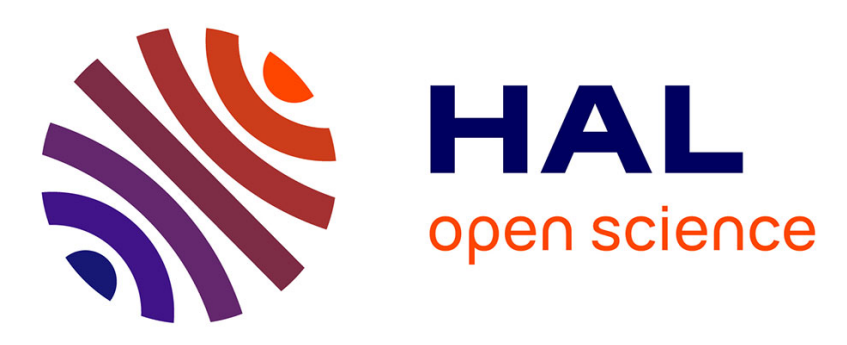

\title{
A color-based selective and interactive filter using weighted TV
}

Cédric Loosli, François Lecellier, Stéphanie Jehan-Besson, Jonas Koko

\section{To cite this version:}

Cédric Loosli, François Lecellier, Stéphanie Jehan-Besson, Jonas Koko. A color-based selective and interactive filter using weighted TV. 15th Conference on Computer Analysis of Images and Patterns, Aug 2013, York, United Kingdom. pp.315-323, 10.1007/978-3-642-40246-3_39 . hal-00860082

\section{HAL Id: hal-00860082 https://hal.science/hal-00860082}

Submitted on 10 Sep 2013

HAL is a multi-disciplinary open access archive for the deposit and dissemination of scientific research documents, whether they are published or not. The documents may come from teaching and research institutions in France or abroad, or from public or private research centers.
L'archive ouverte pluridisciplinaire HAL, est destinée au dépôt et à la diffusion de documents scientifiques de niveau recherche, publiés ou non, émanant des établissements d'enseignement et de recherche français ou étrangers, des laboratoires publics ou privés. 


\title{
A color-based selective and interactive filter using weighted TV
}

\author{
C. Loosli ${ }^{1}$, F. Lecellier ${ }^{2}$, S. Jehan-Besson ${ }^{3}$, and J. Koko ${ }^{1}$ \\ 1 LIMOS, Université Blaise Pascal - CNRS UMR 6158, Complexe des Cézeaux, 63173 Aubière, France \\ 2 XLIM-SIC, UMR CNRS 7252, Bd Marie et Pierre Curie BP 30179, 86962 Futuroscope Chasseneuil cedex, France \\ 3 GREYC Laboratory, CNRS UMR 6072, Bd du Maréchal Juin, 14050 Caen, France
}

\begin{abstract}
In this paper we propose to introduce a new color interactive and selective filtering tool based on the minimization of a weighted vectorial total variation term $T V_{g}$ with the $L^{2}$ norm as data term. Our goal is to filter one region of an image while preserving the other using a minimal interaction with the user. To this end, we take benefit of a weighted vectorial regularization term $T V_{g}$ based on color moments in order to perform our selective color filtering. Up to now, color moments have been mainly introduced for indexation purposes. In our case, the user selects some points in the area to preserve and some other points in the area to be filtered. Reference color moments are then computed on patches around the selected points and are included in the $T V_{g}$ term through various functions $g$. Two main $g$ functions are tested within the HSL color space leading to very interesting results on both synthetic and real images. Convex optimization tools are then used to solve the minimization issue. An augmented Lagrangian formulation leads to a simple and efficient algorithm based on Uzawa block relaxation schemes. Our algorithm, while easy to be implemented, proves to be efficient in terms of computational cost due to its robustness towards the choice of the penalty parameter. The proposed filtering tool may be very interesting as a pre-processing step for segmentation, movie post-production or object-oriented compression.
\end{abstract}

\section{Introduction}

In many image processing issues, a filtering step is required in order to remove noise or spurious details from the initial image. The image may then be decomposed in regions (e.g. homogeneous regions) using a segmentation algorithm, or some regions of interest may be selected using well-defined algorithms (active contours for example). The interactive color filtering tool proposed in this paper is quite different from a simple filtering step and may even be considered as a pre-segmentation algorithm. Indeed, the main idea is to filter and simplify some parts of the image while preserving some others using some color properties of the regions. In order to obtain such a filtering tool, a variational framework is settled. We focus on the minimization of functionals that take benefit of a color weighted total variation $\left(T V_{g}\right)$ regularization term coupled with the minimization of a $L^{2}$ data term. The total variation $(T V)$ regularization term owns some interesting geometrical properties $[19,1,6]$ that can be nicely used for denoising and segmentation. The $T V$ regularization term was first proposed for denoising in [20] coupled with the minimization of the $\mathbf{L}^{\mathbf{2}}$ norm as a data term (the well-known ROF model). More recently, Bresson et al [1] proposed to introduce a spatially adaptive TV term while using a function $g$ inside the integral of the $T V$ term. The function $g$ allows to take into account the image gradient in the $T V$ term in order to preserve object boundaries during the denoising process. Some other authors also take benefit of such a weighted TV [5, 13, 1, 14, 16, 17].

In this paper, we propose to settle an interactive color image filtering algorithm by introducing color moments in the weighted TV regularization term. The user is expected to draw a curve on the region to be removed and another curve on the region to be preserved. The color moments of the chosen points are then computed on small patches and the corresponding characteristics are included in a well-adapted $g$ function in the $T V_{g}$ term. As far as color moments are concerned, they are considered as a powerful color descriptor. 
They have been introduced in [18] for image indexation and retrieval in order to complete or replace SIFT descriptors. They appear to be more interesting than the classical color histograms used in $[9,11,15]$. Indeed, color histograms are not able to represent the spatial layout of the color repartition. In an original manner, we propose to take advantage of such a representation for our selective color filtering tools. Concerning the optimization of the whole functional $\left(T V_{g}+L^{2}\right)$, we rely on the mathematical framework introduced in [16] for grey level images. We propose to tackle the optimization of the vectorial $T V_{g}+L^{2}$ using an augmented Lagrangian approach and Uzawa block relaxation schemes. Our numerical scheme presents the advantage to be robust regarding with the choice of the penalty parameter and is efficient in terms of computational cost. The proposed filtering tool may be very interesting as a pre-processing step for segmentation, movie post-production or object-oriented compression.

In the rest of the paper, we first detail the main principles of our selective filtering tool in section 2 . Then we propose to give the main lines of the optimization process in section 3. The color-based weighted TV is introduced in section 4. Finally some experimental results on both synthetic and real images are displayed in order to check the availability of our tool.

\section{Selective filtering based on the minimization of a weighted TV}

\subsection{Geometrical properties of TV and weighted TV}

The classical ROF (Rudin, Osher and Fatemi) model [20] aims to recover the original image $u(x)$ given a noisy image $f(x)$ by minimizing the total variation under $L^{2}$ data fidelity:

$$
E(u)=\int_{\Omega}|\nabla u(x)| d x+\lambda \int_{\Omega}(u(x)-f(x))^{2} d x,
$$

where $\Omega \subset \mathbb{R}^{2}$, is the image domain and $\lambda$ a positive scale parameter.

The first integral is the classical regularization term called TV (Total Variation) term. This term has proven its efficiency for image restoration and also presents some interesting geometrical properties. In order to better explain these properties, let us denote the upper level sets of the image by $U^{\alpha}(u)=\{x, u(x)>\alpha\}$ like in [4]. From a geometrical point of view, the co-area formula [8] states that, for any function which belongs to the space of bounded variations $\operatorname{BV}(\Omega)$, there is a relation between the TV regularization term and the perimeter $\operatorname{Per}\left(U^{\alpha}\right)$ of the set $U^{\alpha}$. Indeed, we can write $\operatorname{Per}\left(U^{\alpha}(u)\right)=\int_{\Omega}\left|\nabla \chi_{U^{\alpha}(u)}\right| d x$ for all $\alpha$ where $\chi_{U^{\alpha}}(u)$ stands for the characteristic function of the set $U^{\alpha}(u)$. Such geometrical features may contribute to explain the properties of this regularization term. Indeed, when decreasing the weight $\lambda$ of the data term, components will be removed in an order determined by their size and their geometry. For example, small components will be removed first and sharp angles will be smoothed. In [6], the authors establish a connection between this model and morphological operators such as opening and morphological granulometry.

The introduction of a $g$ function in TV may produce different filtering results. Indeed, the $T V_{g}$ term, when applied to a characteristic set is equivalent to a weighted perimeter $\int_{C} g(s) d s$ where $C$ designates the boundary of the set and $s$ its arc length. In [1], the function $g$ is then chosen as $g(x)=1 /\left(1+\beta G_{\sigma} *|\nabla f|\right)$ in order to introduce the image gradient directly in the regularization term. This term allows to preserve object boundaries and sharp angles during the regularization process and can also be used for shape segmentation [1]. Indeed, this regularization term corresponds to the classical criterion proposed in geodesic active contours [2]. Note that such a spatially varying TV term has also been investigated by different authors with various $\mathrm{g}$ functions (e.g. for salt and pepper denoising) [5, 13, 1, 14, 16, 17]. 


\subsection{Color selective filter using weighted TV}

In this paper, we propose to test the availability of the weighted TV regularization term in order to perform a kind of selective filtering of the image components. This idea is closely related to the design of geometrical filters in the framework of mathematical morphology where some shapes are removed on the basis of their geometric properties. Some first examples of a geometrical filtering using the $T V_{g}$ term are given in $[16,17]$. Rather than using geometric properties, we here propose to take benefit of color moments and of a vectorial TV term in order to include some color features in the function $g$. In order to introduce a selective filtering scheme, we add an interactive step where two regions are manually selected (using a curve drawn on each region). Then the color moments of each point within each region are computed leading to a reference vector of moment for each region. The function $g$ is then designed in order to filter one region while preserving the other one and inversely. The main principle of this scheme is shown in Fig.1.

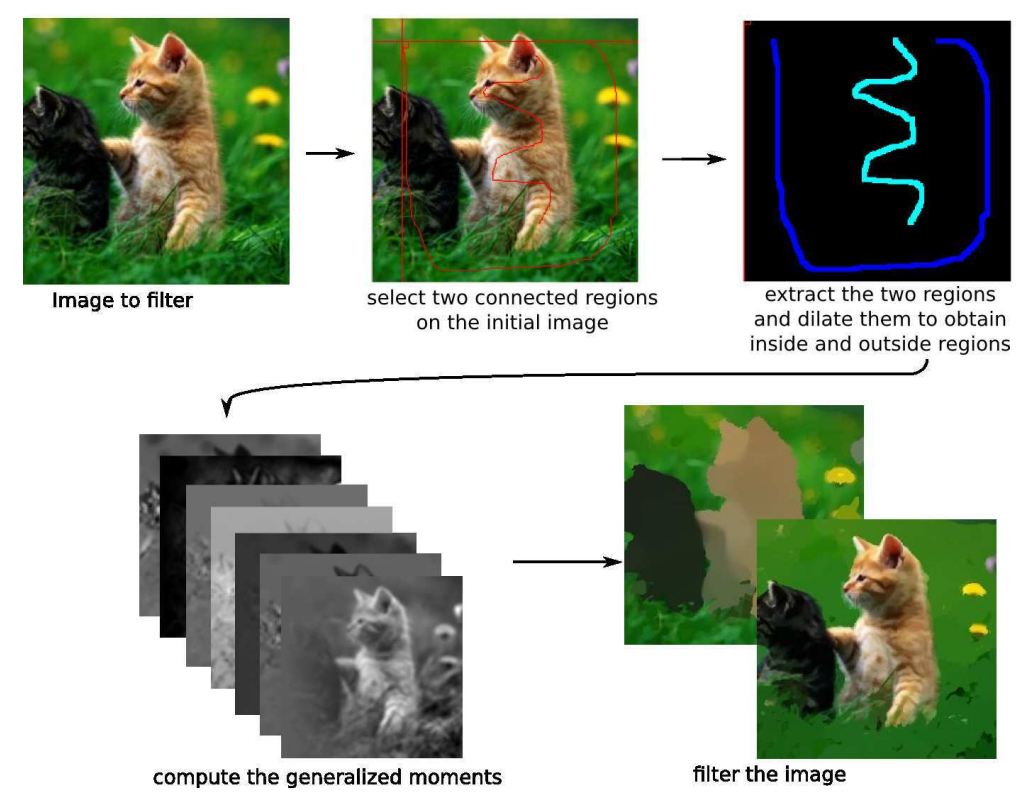

Fig. 1. Main principles of the proposed color interactive and selective filtering tool.

\section{Fast dual minimization of $T V_{g}+L^{2}$}

Let $\Omega$ be a three-dimensional bounded open domain of $\mathbb{R}^{d}, d=2,3$ and a vector-valued function $\mathbf{u}(x)=$ $\left(u_{1}(x), u_{2}(x), u_{3}(x)\right) \in \mathbb{R}^{3}$ defined on $\Omega$ that corresponds to the color intensity with the three values of each color channel. In the rest of the document, vector valued functions are denoted by bold-face letters (e.g. $\left.\mathbf{u}=\left(u_{1}, u_{2}, u_{3}\right)\right)$. Let us also note the Euclidean scalar product by $\mathbf{u} \cdot \mathbf{v}=\sum_{i=1}^{3} u_{i} v_{i}$, for $\mathbf{u}$ and $\mathbf{v}$ in $\mathbb{R}^{d}$. Moreover, for $\mathbf{u} \in \mathbf{R}^{d}$, we use the notation $|\mathbf{u}|_{2}=(\mathbf{u} \cdot \mathbf{u})^{1 / 2}$ for the Euclidean norm.

Let $g$ be a continuous, positive valued and bounded function defined on $\Omega$, when dealing with color images, we consider the following weighted total variation regularization term, denoted by $T V_{g}$ :

$$
T V_{g}(\mathbf{u})=\int_{\Omega} g(x)\left[\left|\nabla u_{1}\right|_{2}^{2}+\left|\nabla u_{2}\right|_{2}^{2}+\left|\nabla u_{3}\right|_{2}^{2}\right]^{1 / 2} \mathrm{~d} x
$$


The considered function $g$ is described later in section 4 .

Let $\mathbf{f}=\left(f_{1}, f_{2}, f_{3}\right)$ be the input color image to be filtered, we propose to address the following vectorial $T V_{g}+L^{2}$ minimization problem:

$$
\min _{\mathbf{u} \in \mathbf{X}} E(\mathbf{u})=J(\mathbf{u})+\lambda \int_{\Omega}|\mathbf{u}(x)-\mathbf{f}(x)|_{2}^{2} \mathrm{~d} x .
$$

where $\mathbf{u}$ is the unknown image to restore and $\mathbf{X}$ is a suitable functions space.

\subsection{Augmented Lagrangian methods for the $T V_{g}+L^{2}$ model}

In this section we propose to use convex optimization tools and dual approaches in order to solve (2). To this end, we need to transform the convex minimization problem (2) into a suitable saddle-point problem by introducing an auxiliary unknown as for the scalar case [16]. An augmented Lagrangian approach is then introduced and solved using Uzawa relaxation schemes. Some details are given thereafter for the reader convenience but only briefly for space reasons.

Let us introduce the auxiliary unknown $\mathbf{p}=\mathbf{f}-\mathbf{u}$ and rewrite the functional $E$ as

$$
E(\mathbf{u}, \mathbf{p})=T V_{g}(\mathbf{u})+\lambda \int_{\Omega}|\mathbf{p}(x)|_{2}^{2} \mathrm{~d} x .
$$

The minimization problem (3) becomes $\min _{(\mathbf{u}, \mathbf{p}) \in K} E(\mathbf{u}, \mathbf{p})$,, where the constraint set $K$ is defined by $K=$ $\{(\mathbf{u}, \mathbf{p}) \in X \times X \mid \mathbf{u}+\mathbf{p}-\mathbf{f}=0$ in $\Omega\}$. It is obvious that the two minimization problems are equivalent. With this constrained minimization problem, we associate the Lagrangian functional $\mathscr{L}$ defined on $\mathbf{X}^{3}$ by

$$
\mathscr{L}(\mathbf{u}, \mathbf{p} ; \mathbf{s})=E(\mathbf{u}, \mathbf{p})+(\mathbf{s}, \mathbf{u}+\mathbf{p}-\mathbf{f})_{\mathbf{X}} .
$$

In (4), $\mathbf{s}$ is the Lagrange multiplier associated with the constraint in $K$. Since $E$ is convex and continuous and the constraint in $K$ is linear, a saddle point $\left(\mathbf{u}^{*}, \mathbf{p}^{*} ; \mathbf{s}^{*}\right) \in \mathbf{X}^{3}$ of $\mathscr{L}$ exists and verifies $\mathscr{L}\left(\mathbf{u}^{*}, \mathbf{p}^{*} ; \mathbf{s}\right) \leq$ $\mathscr{L}\left(\mathbf{u}^{*}, \mathbf{p}^{*} ; \mathbf{s}^{*}\right) \leq \mathscr{L}\left(\mathbf{u}, \mathbf{p} ; \mathbf{s}^{*}\right), \quad \forall(\mathbf{u}, \mathbf{p}, \mathbf{s}) \in \mathbf{X}^{3}$.

We now introduce the augmented Lagrangian defined, for $r>0$, by

$$
\mathscr{L}_{r}(\mathbf{u}, \mathbf{p} ; \mathbf{s})=\mathscr{L}(\mathbf{u}, \mathbf{p} ; \mathbf{s})+\frac{r}{2}\|\mathbf{u}+\mathbf{p}-\mathbf{f}\|_{L^{2}}^{2}
$$

where $r$ is the penalty parameter. We then consider the following saddle-point problem

$$
\mathscr{L}_{r}\left(\mathbf{u}^{*}, \mathbf{p}^{*} ; \mathbf{s}\right) \leq \mathscr{L}_{r}\left(\mathbf{u}^{*}, \mathbf{p}^{*} ; \mathbf{s}^{*}\right) \leq \mathscr{L}_{r}\left(\mathbf{u}, \mathbf{p} ; \mathbf{s}^{*}\right), \quad \forall(\mathbf{u}, \mathbf{p}, \mathbf{s}) \in \mathbf{X}^{3} .
$$

It can be proved (easily) that a saddle point of $\mathscr{L}_{r}$ is a saddle point of $\mathscr{L}$ and conversely. This is due to the fact that the quadratic term in $\mathscr{L}_{r}$ vanishes when the constraint $\mathbf{u}+\mathbf{p}-\mathbf{f}=0$ is satisfied. Some efficient numerical schemes can be used to solve this problem like notably the Uzawa Block Relaxation methods detailed thereafter. One important feature is that this algorithm is well-conditioned and robust against the choice of the penalty parameter $r$ [16].

\subsection{Uzawa block relaxation methods}

We apply the following Uzawa block relaxation method to solve the saddle-point (5) by using an alternative minimization procedure (see e.g. $[10,12]$ ). Given $\mathbf{p}^{-1}$ and $\mathbf{s}^{0}$, we compute successively $\mathbf{u}^{k}, \mathbf{p}^{k}$ and $\mathbf{s}^{k+1}$ as 
follows

$$
\begin{aligned}
& \mathbf{u}^{k}=\arg \min _{\mathbf{u}} \mathscr{L}_{r}\left(\mathbf{u}, \mathbf{p}^{k-1}, \mathbf{s}^{k}\right) \\
& \mathbf{s}^{k+1 / 2}=\mathbf{s}^{k}+\frac{r}{2}\left(\mathbf{u}^{k}+\mathbf{p}^{k-1}-f\right) \\
& \mathbf{p}^{k}=\arg \min _{\mathbf{p}} \mathscr{L}_{r}\left(\mathbf{u}^{k}, \mathbf{p}, \mathbf{s}^{k}\right) \\
& \mathbf{s}^{k+1}=\mathbf{s}^{k+1 / 2}+\frac{r}{2}\left(\mathbf{u}^{k}+\mathbf{p}^{k}-f\right)
\end{aligned}
$$

- Solution of subproblem (7) The functional $\mathbf{u} \mapsto \mathscr{L}_{r}\left(\mathbf{u}, \mathbf{p}^{k-1} ; \mathbf{s}^{k}\right)$ can be rewritten as $\Phi_{1}(\mathbf{u}):=\frac{r}{2}\|\mathbf{u}\|_{L^{2}}^{2}$ $+J(\mathbf{u})+(\tilde{\mathbf{p}}, \mathbf{u})_{X}+C$, where $C$ is a constant and $\tilde{\mathbf{p}}=\mathbf{s}^{k}+r\left(\mathbf{p}^{k-1}-\mathbf{f}\right)$. Using Fenchel duality theory (see e.g. [7]), the solution of (7) is $\mathbf{u}^{k}=\left(\nabla \cdot \mathbf{v}^{k}-\tilde{\mathbf{p}}\right) / r$ where $\mathbf{v}^{k}$ is the solution of $\inf _{|\mathbf{v}|_{2}^{2}-g^{2} \leq 0} \frac{1}{2 r}\|\nabla \cdot \mathbf{v}-\tilde{\mathbf{p}}\|_{L^{2}}^{2}$. We can then compute $\mathbf{v}^{k}$ using the following semi-implicit scheme due to Chambolle [3].

$$
v_{i}^{\ell+1}=\frac{v^{\ell}+\tau \nabla\left(\nabla \cdot v_{i}^{\ell}-\tilde{p}_{i}\right)}{1+(\tau / g)\left[\sum_{i=1}^{3}\left|\nabla\left(\nabla \cdot v_{i}^{\ell}-\tilde{p}_{i}\right)\right|_{2}^{2}\right]^{1 / 2}}, \quad i=1,2,3, \quad \text { where } \tau>0
$$

- Solution of subproblem (9) The functional $\mathbf{p} \mapsto \mathscr{L}_{r}\left(\mathbf{u}^{k}, \mathbf{p} ; \mathbf{s}^{k}\right)$ can be rewritten as

$$
\Phi_{2}(\mathbf{p})=(\lambda+r / 2)\|\mathbf{p}\|_{L^{2}}^{2}+\left(\mathbf{s}^{k}+r\left(\mathbf{u}^{k}-\mathbf{f}\right), \mathbf{p}\right)_{X}+C,
$$

where $C$ is a constant. A straightforward calculation yields $\mathbf{p}^{k}=-\left(\mathbf{s}^{k}+r\left(\mathbf{u}^{k}-\mathbf{f}\right)\right) /(r+2 \lambda)$.

- Uzawa block relaxation algorithm With the results above, we can now present the Uzawa block relaxation algorithms for the $T V_{g}+L^{2}$ model. The procedure is detailed in Algorithm 1. We iterate until relative error on $\left(\mathbf{u}^{k}, \mathbf{p}^{k}\right)$ becomes sufficiently small.

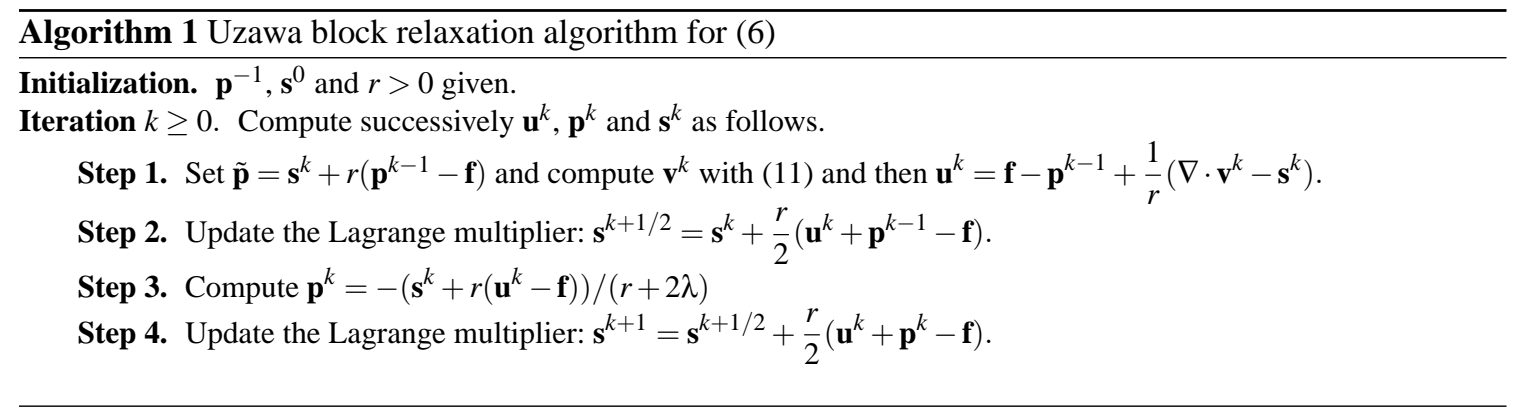

Such algorithms are fast, easy to implement, and also robust to the choice of the penalty parameter which allows to choose a value of $r$ that minimizes the number of iterations and so the computational cost [17].

\section{Proposition of a weighted TV based on Color moments}

Let us now introduce the weighted TV regularization designed for our color selective filtering tool. Given a color image $\mathbf{u}=\left(u_{1}, u_{2}, u_{3}\right)$, we propose to take benefit of generalized color moments $M_{p q}^{a b c}$ introduced in 
[18]. The color moment of order $p+q$ and degree $a+b+c$ is defined as follows:

$$
M_{p q}^{a b c}=\iint_{D} x_{1}^{p} x_{2}^{q}\left[u_{1}\left(x_{1}, x_{2}\right)\right]^{a}\left[u_{2}\left(x_{1}, x_{2}\right)\right]^{b}\left[u_{3}\left(x_{1}, x_{2}\right)\right]^{c} d x_{1} d x_{2},
$$

where $x=\left(x_{1}, x_{2}\right)$ is a pixel in a 2D image and $a, b, c, p$ and $q$ some positive integers and $D$ the domain of computation of the moment (it can then be the whole image or a patch).

In order to use a semi-local information on color (which can be really interesting for texture regions), we propose to compute color moments on small patches around each pixel of the considered region. We denote this small neighborhood by $D_{n}$ where $n$ represents the half size of the neighborhood. Note that the moments of degree 0 correspond simply to the classical geometric moments of $D_{n}$ and so they do not provide useful information for filtering since they only encode the fixed geometry of $D_{n}$. In our application we consider 27 moments in order to reduce the computational time which are $M_{00}^{a b c}, M_{01}^{a b c}$ and $M_{10}^{a b c}$ with $(a, b, c) \in\{(1,0,0) ;(0,1,0) ;(0,0,1) ;(1,1,0) ;(1,0,1) ;(0,1,1) ;(2,0,0) ;(0,2,0) ;(0,0,2)\}$. The corresponding 27-vectors of moments are noted $\mathbf{M}(x)$ where $x \in \Omega$. In our application we consider the HSL color space for the computation which conducts in the practice to better results than the RGB color space. Note that the space color can be changed easily using a simple conversion at the beginning of the process.

The semi-supervised filtering scheme has been previously described in Figure 1. In the first step, we draw two curves on the image, one for the "inside" region and the other for the "outside" region. The "outside" region $\Omega_{\text {out }}$ will be filtered and the "inside" one $\Omega_{\text {in }}$ remains without filtering. A small dilatation of what we can call "the markers" is performed and the color moments for each selected region are then computed. We present there two main solutions for the design of a weighted TV term taking into account these color moments.

\subsection{Definition of weighted TV based on the average moment vector}

The most straightforward method is to compute an average moment vector of the markers $\Omega_{i n}$ and $\Omega_{\text {out }}$ (averaging each component of the vector). The average value for the component $\alpha$ is $\mu_{i}^{\alpha}=\frac{1}{\left|\Omega_{i}\right|} \int_{\Omega_{i}} M_{\alpha}\left(x_{1}, x_{2}\right) d x_{1} d x_{2}$ with $\alpha \in[0,27]$ and $i=$ in or out. We then propose to select the order $\alpha^{*}$ that corresponds to the maximum distance between the two average values as follows :

$$
\alpha^{*}=\max _{\alpha \in[0,27]}\left(\left|\left(\mu_{\text {in }}^{\alpha}-\mu_{\text {out }}^{\alpha}\right)\right|\right),
$$

We then propose to introduce the following thresholding function :

$$
T(x)=\left\{\begin{array}{l}
c_{1} \text { if } M_{\mathrm{Q}^{*}}(x)<\mu_{\mathrm{Q}^{*}} \\
c_{2} \text { if } M_{\mathrm{Q}^{*}}(x) \geq \mu_{\mathrm{Q}^{*}}
\end{array}\right.
$$

where $\mu_{\alpha^{*}}$ corresponds to the average of the moments $M_{\alpha^{*}}$ of order $\alpha^{*}$ of the whole image $\Omega$ and is then chosen as a threshold. We choose $c_{1}=0.01$ and $c_{2}=1$ or inversely in order to uppermost smooth the pixels corresponding to the reference region $r_{\text {out }}$ or $r_{i n}$. A regularized continuous version of the function $T(x)$ is needed which is obtained by a Gaussian filtering: $g(x)=G_{\sigma} * T(x)(\sigma=0.05)$.

\subsection{Definition of a weighted TV using $1-n n$ selection}

The previous method is not able to deal with large variations of color inside each reference region. In order to cope with this problem, we propose to consider the whole 27 vector as a descriptor. We then compute the 
$L_{2}$ distance between the 27 moments of pixel $\mathbf{x}$ and the 27 moments of each pixel of the inside and outside region and we select the nearest pixel in each reference region. So, for each pixel $x$ of the image, we choose the pixel $\mathbf{x}^{*}$ in the reference region $r_{i}(i=$ in or out $)$ such that : $x_{i}^{*}=\arg \min _{\mathbf{y} \in r_{i}}\left[\sum_{\alpha}\left(M^{\alpha}(x)-M^{\alpha}(y)\right)^{2}\right]$. For each pixel $x$, the pixel $x_{i}^{*}$ designates the pixel in the region $r_{i}$ with the nearest moment values to $x$ in the sense of the $L^{2}$ norm.

A thresholding function is then defined using $T(x)=c_{1}$ if $x_{i n}^{*} \in r_{i n}$ and $c_{2}$ if $x_{i n}^{*} \in r_{\text {out }}$. We choose $c_{1}=0.01$ and $c_{2}=1$ in order to uppermost smooth the pixels corresponding to the reference region $r_{\text {out }}$ while preserving $r_{i n}$. The role of $r_{i n}$ and $r_{\text {out }}$ can be inverted to preserve $r_{\text {out }}$. A regularized continuous version of the function $T\left(x\right.$ is needed which is obtained by a Gaussian filtering: $g(x)=G_{\sigma} * T(x)(\sigma=0.05)$.

\section{Experimental results}

To test our approach, we conduct tests on synthetic and natural images using the two different $g$ functions $(\mu$ and $1-n n)$. The patch size is chosen as $n=3$ which represents a good trade-off between precision and quantity of information (a higher patch size can be needed in order to filter texture with larger patterns). The results are depicted in Fig.2 and Fig.3. In each figure, we both display the two results obtained when choosing $r_{\text {in }}$ or $r_{\text {out }}$ to be preserved.

The comparison between $\mu$ and $1-n n$ is straightforward. The $1-n n$ method outperforms significantly the other one showing the interest of designing a well-adapted function $g$. This can be easily explain since $\mu$ only compares the average of the whole selection with the current moment of the pixel. The $1-n n$ method selects interactively the nearest vector of moments inside each region and then the nearest region (inside or outside) to apply the filtering. So it delivers a more local descriptor of the selected regions.

Since the results give a strong smoothing effect on the outside region while preserving all the details of the inside one, this method gives a straightforward way to perform an object-oriented compression. When considering the "cats" image, a classic JPEG compression with a factor of 0.9 leads to an image of size $25 \mathrm{~Kb}$. The same JPEG compression on the filtered image where the two cats are selected and the background is smoothed leads to an image size of $17 \mathrm{~Kb}$. So, depending on the size of the region of interest, our filtering method may compress the images with a ratio from $30 \%$ to $80 \%$ while preserving some selected components. The algorithm can also be used as a pre-processing step for different applications such as segmentation or object selection or even artistic post-production of images or movies.

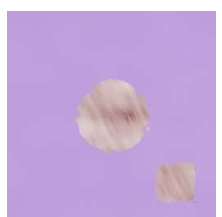

(a)

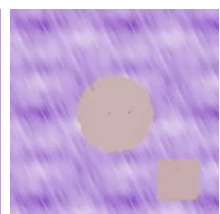

(b)

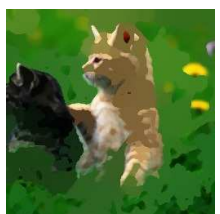

(c)

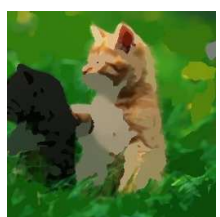

(d)

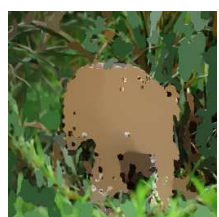

(e)

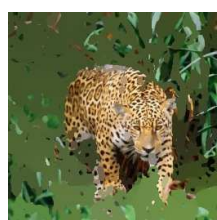

(f)

Fig. 2. Experimental results on a synthetic image and on two natural images (selection and filtering of the background in (a) and of the geometric components in (b), selection and filtering of the two cats in (c) and of the background in (d), selection and filtering of the leopard in (e) and of the background in (f)) using $\mu$ approach and HSL space. 


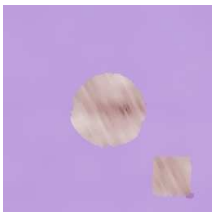

(a)

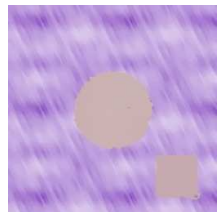

(b)

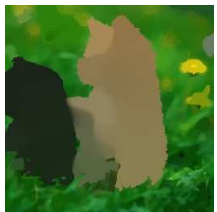

(c)

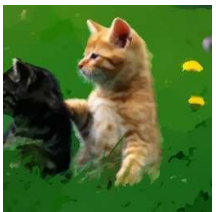

(d)

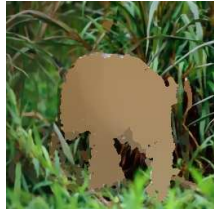

(e)

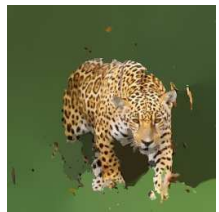

(f)

Fig. 3. Experimental results on a synthetic image and on two natural images (selection and filtering of the background in (a) and of the geometric components in (b), selection and filtering of the two cats in (c) and of the background in (d), selection and filtering of the leopard in (e) and of the background in (f)) using $1-n n$ approach and HSL space.

\section{References}

1. Xavier Bresson, Selim Esedoglu, Pierre Vandergheynst, Jean-Philippe Thiran, and Stanley Osher. Fast global minimization of the active contour/snake model. JMIV, 28:151-167, 2007.

2. V. Caselles, R. Kimmel, and G. Sapiro. Geodesic active contours. IJCV, 22:61-79, 1997.

3. A. Chambolle. An algorithm for total variation minimization and applications. JMIV, 20(1-2):89-97, 2004.

4. J. Darbon and M. Sigelle. Image restoration with discrete constrained total variation part I: Fast and exact optimization. JMIV, 26(3):261-271, 2006.

5. Y. Dong, M. Hintermüller, and M. Montserrat Rincon-Camacho. A multi-scale vectorial $\mathrm{L}^{\tau}$-TV framework for color image restoration. IJCV, 92:296-307, 2011.

6. V. Duval, J. Aujol, and Y. Gousseau. The TV L1 model: a geometric point of view. Multiscale Model. Simul, 8:154-251, 2009.

7. I. Ekland and R. Temam. Convex Analysis and Variational Problems. SIAM, Philadelphia, 1999.

8. W. Fleming and R. Rishel. An integral formula for total gradient variation. Archiv der Mathematik, 11:218-222, 1960.

9. D. Forsyth. A novel algorithm for color constancy. Journal of Computer Vision, pages 5-36, 1990.

10. M. Fortin and R. Glowinski. Augmented Lagrangian Methods: Application to the Numerical Solution of Boundary-Value Problems. North-Holland, Amsterdam, 1983.

11. B. Funt and G. Finlayson. Color constant color indexing. IEEE PAMI, pages 522-529, 1995.

12. R. Glowinski and P. Le Tallec. Augmented Lagrangian and Operator-splitting Methods in Nonlinear Mechanics. SIAM, Philadelphia, 1989.

13. B. Goldlücke and D. Cremers. An approach to vectorial total variation based on geometric measure theory. In CVPR, volume 106, pages 327-333, 2010.

14. M. Grasmair. Locally adaptive total variation regularization. In SSVM, pages 331-342, 2009.

15. G. Healey and D. Slater. Using illumination invariant color histogram descriptors for recognition. In Computer Vision and Pattern Recognition, pages 355-360, 1994.

16. Jonas Koko and Stéphanie Jehan-Besson. An Augmented Lagrangian Method for $\mathrm{TV} g+\mathrm{L}^{1}$-norm Minimization. JMIV, 38(3):Pages 182-196, November 2010.

17. C. Loosli, S. Jehan-Besson, and J. Koko. Uzawa block relaxation methods for color image restoration. In $\underline{\mathrm{CPCV}}$ Workshop in ECCV, pages 492-503, 2012.

18. Florica Mindru, Tinne Tuytelaars, Luc Van Gool, and Theo Moons. Moment invariants for recognition under changing viewpoint and illumination. Computer Vision and Image Understanding, pages 3-27, 2004.

19. Mila Nikolova, Selim Esedoglu, and Tony F. Chan. Algorithms for finding global minimizers of image segmentation and denoising models. SIAM Journal of Applied Mathematics, 66(5):1632-1648, 2006.

20. L. Rudin, S. Osher, and E. Fatemi. Nonlinear total variation based noise removal algorithms. Physica D., 60:259$268,1992$. 